\title{
Phenomenon of Mobility in Non-Linear Theories ${ }^{\star}$
}

\author{
Bogdan Mielnik ${ }^{\star}$ \\ Departamento de Fìsica, CINVESTAV, Apdo. Postal 14-740, 07000, México D.F.
}

\begin{abstract}
Homeomorphisms of the unit-sphere of states are studied. Generalizations of the Piron statement and Wigners' theorem are obtained. It is shown that if the semigroup of the unitary operations of quantum theory were extended by introducing any non-linear operation, a mobility phenomenon would occur consisting of a possibility of moving any two states to any two surroundings on the unit sphere. For the resulting "non-linear wave packets" the complementarity doctrine would become impossible because of "fluidity" of the space of states under the dynamical transformations.
\end{abstract}

\section{Introduction}

In one of its present day views, the theory of dynamical systems deals with the iterations of one single transformation $U: \Phi \rightarrow \Phi$ of a certain manifold of states $\Phi$. Within this line of thought some interesting results have been achieved, such as the existence of the "strange attractors," etc. [1-3]. However, the approach based merely on one operation means a simplification of a dynamical theory ad extremis. The real physical system is submerged in a variable universe; by modifying it, one can induce many distinct evolution operations. Hence, a complete dynamical theory should deal not with one but with many alternative operations [4-8]. In the "multi-operation dynamics" some new questions arise. One of them is: what kind of transformations of the manifold of states $\Phi$ can be basically achieved by manipulating the operations available? [7-9]. In the dynamical theories of quantum mechanical character, the answer is basically known. Here, the evolution operations are unitary transformations of the unit sphere in a Hilbert space. Provided that there are no superselection rules, all the unitary transformations can be principally achieved by employing adequate external fields $[7,10,11]$. A qualitatively new phenomenon arises when the idea of multi-operational dynamics is associated with non-linear evolution equations.

* Supported by COSNET/SEP

$\star \star$ On leave of absence from Institute of Physics, Warsaw University, Warsaw, Poland 


\section{Mobility Phenomenon (A Review)}

It might seem that when a little non-linear term is added to the conventional quantum mechanical equations of evolution, the resulting theory will differ "little" from that based on the unitary operations. However, in a sequence of models, once the linearity is lost, a qualitative metamorphosis occurs.

In the two dimensional analogue of non-linear wave mechanics [8] the hypothetical "states" are rays of 2-component complex vectors $\psi=\left\|\psi_{j}\right\|_{j=1,2}$ obeying the evolution equation:

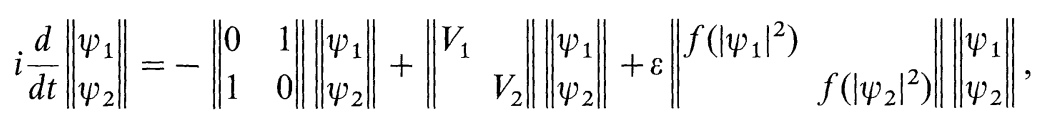

where $V_{1}, V_{2} \in \mathbb{R}$ imitate an "external potential" and $f(\cdot)$ is a given function. The normalization condition conserved by the motion (2.1) is $\left|\psi_{1}\right|^{2}+\left|\psi_{2}\right|^{2}=1$. By introducing the real coordinates $x=\psi_{1} \bar{\psi}_{2}+\psi_{2} \bar{\psi}_{1}, y=i\left(\psi_{1} \bar{\psi}_{2}-\psi_{2} \bar{\psi}_{1}\right), z=\left|\psi_{1}\right|^{2}$ $-\left|\psi_{2}\right|^{2}$, with

$$
x^{2}+y^{2}+z^{2}=\left(\left|\psi_{1}\right|^{2}+\left|\psi_{2}\right|^{2}\right)^{2}=1,
$$

one maps the rays into the points of the unit sphere $S$ in $\mathbb{R}^{3}$; the orthogonal rays being represented by the antipodes of $S$. Now, when $\varepsilon=0$ (linear case) the evolution operations obtained by integrating (2.1) for all possible time dependent $V_{1}, V_{2}$ yield all the unitary transformations of the $\psi$-vectors, geometrically represented by the rigid rotations of the sphere $S$. When $\varepsilon \neq 0$, the picture suddenly changes. The operations defined by (2.1) still allows us to achieve the rigid rotations as the limiting cases. However, the semigroup of the evolution operations contains now a family of non-linear, distance-changing homeomorphisms of the sphere. This family is so rich that there is no limitation for the possibility of changing distances: for any two $x, y \in S, x \neq y$, and any two open neighborhoods $\Omega, \Omega^{\prime} \subset S$, there are evolution operations which simultaneously bring $x$ and $y$ to $\Omega$ and $\Omega^{\prime}$. In particular, any two different points $x, y \in S$, no matter how close, can be separated at will, by bringing them to the vicinities of any two antipodes of $S$ (orthogonal states). This is what we call a mobility phenomenon.

An almost exact repetition of this phenomenon has been detected for a different two component model:

$$
i \frac{d}{d t}\left\|\begin{array}{c}
\Psi_{1} \\
\psi_{2}
\end{array}\right\|=-\left\|\begin{array}{cc}
0 & \left|\psi_{2}\right|^{2} \\
\left|\psi_{1}\right|^{2} & 0
\end{array}\right\|\left\|\begin{array}{c}
\psi_{1} \\
\psi_{2}
\end{array}\right\|+\left\|\begin{array}{cc}
V_{1} & \\
& V_{2}
\end{array}\right\|\left\|\begin{array}{c}
\psi_{1} \\
\psi_{2}
\end{array}\right\| ; \quad V_{1}, V_{2} \in \mathbb{R} .
$$

Here, the basic conservation law is $\left|\psi_{1}\right|^{4}+\left|\psi_{2}\right|^{4}=$ const, suggesting the invariant normalization $\left|\psi_{1}\right|^{4}+\left|\psi_{2}\right|^{4}=1$, and giving a hint that $\left|\psi_{1}\right|^{4}$ and $\left|\psi_{2}\right|^{4}$ might play the role of "quantum probabilities". ${ }^{1}$ ) As before, the states are rays. By introducing new real coordinates: $x=\frac{1}{2}\left(\psi_{1} \bar{\psi}_{2}+\psi_{2} \bar{\psi}_{1}\right), \quad y=\frac{i}{2}\left(\psi_{1} \bar{\psi}_{2}-\psi_{2} \bar{\psi}_{1}\right)$,

1 As can be easily seen, Eqs. (2.1) and (2.2) are the analogues of the non-linear wave equations: $i \frac{\partial \psi}{\partial t}=-\frac{1}{2} \Delta \psi+V \psi+\varepsilon f\left(|\psi|^{2}\right) \psi$ and $i \frac{\partial \psi}{\partial t}=-\frac{1}{2} \Delta\left(|\psi|^{2} \psi\right)+V \psi$, whose conservative integrals are $\int_{\mathbb{R}^{3}}|\psi|^{2} d_{3} x$ and $\int_{\mathbb{R}^{3}}|\psi|^{4} d_{3} x$ respectively (see $[6]$ ) 


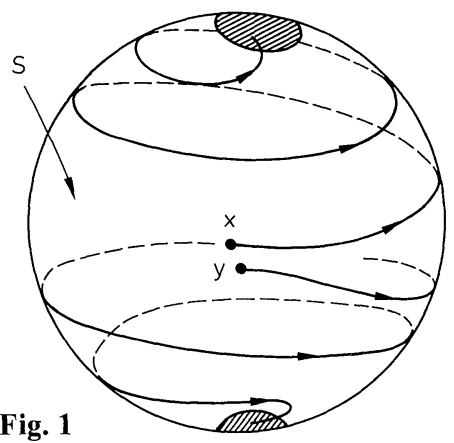

$z=\left|\psi_{1}\right|^{2}-\left|\psi_{2}\right|^{2}$ with $x^{2}+y^{2}+z^{2}=\left|\psi_{1}\right|^{4}+\left|\psi_{2}\right|^{4}=1$, one sees that the set of states is again a unit sphere in $\mathbb{R}^{3}$. By looking at (2.2), one sees now that the relation pointantipode is motion invariant (due to the invariance of (2.2) under the reflection $\left.\psi_{1} \rightarrow-\bar{\psi}_{2}, \psi_{2} \rightarrow \bar{\psi}_{1}\right)$. However this is the only exception from the general "fluidity" of the states: any two states which are not in the point antipode relation can drift simultaneously to any two vicinities on $S$ (authors notes, Geneve 1977, unpublished).

The above "mobility phenomenon" is not exclusive for two component models. The lattice imitations of the wave packets obeying a non-linear analogue of the Schrödinger equation exhibit a similar effect [8]. For genuine wave functions a different but related effect has been observed by Haag and Bannier [12]. The techniques used in [12] were recently applied to a wider class of non-linear wave equations [9]. These results suggest that the phenomenon outlined above is not accidental. After looking at the examples presented, one can see, in fact, that they have a certain common feature: in all of them the semigroup of all dynamical operations is not unitary, but it contains the unitary group either as a subgroup or as a limiting case. We shall show that this is a decisive circumstance: for any essentially non-linear semigroup with that property a similar" phenomenon of mobility" must occur.

\section{Limiting and Conservative Distances}

Below, $S$ denotes the unit sphere in a real or complex Hilbert space $\mathscr{H}$,

$$
S=\{\psi \in \mathscr{H}:\|\psi\|=1\},
$$

and $\Phi$ the projective unit sphere (the set of all unit ray in $\mathscr{H}$ ). The vectors of $S$ will be denoted $\psi, \phi, \ldots$, while the corresponding rays (equivalence classes of vectors) $\{\psi\},\{\phi\} \ldots$ Two rays $a=\{\psi\}, b=\{\phi\}$ will be said to be orthogonal $(a \perp b)$ if $(\psi, \phi)=0$. For any two rays $a, b$ the angular distance $\delta(a, b)$ will be defined by:

$$
\delta(a, b)=\arccos |(\psi, \phi)| .
$$

As is easily seen, $\delta(a, b)$ makes $\Phi$ a complete, metrical space with the topology equivalent to that induced by the original topology of $\mathscr{H}$. If $\mathscr{H}$ is real, the angular 
distance can be also defined for any two vectors $\psi, \phi \in S$, as $\gamma(\psi, \phi)=\arccos (\psi, \phi)$ and varies in $[0, \pi]$. Obviously, for $a=\{\psi\}, b=\{\phi\}, \delta(a, b)=\gamma(\psi, \phi)$ if $\gamma(\psi, \phi) \leqq \frac{\pi}{2}$, while $\delta(a, b)=\pi-\gamma(\psi, \phi)$ if $\gamma(\psi, \phi) \geqq \frac{\pi}{2}$.

A case of a multi-operational dynamics will now be obtained by taking $\Phi$ as the manifold of states and by assuming the existence of a certain distinguished semigroup $G$ of available dynamical transformations of $\Phi$ (the "mobility semigroup" [8]). We shall assume that the elements $g \in G$ are homeomorphisms of $\Phi$. The topology in $G$ will be introduced as the topology of pointwise convergence for the mappings $g: \Phi \rightarrow \Phi$. Simultaneously, by $S U(\Phi)$ we shall denote the group of transformations $\Phi \rightarrow \Phi$ generated by the special unitary transformations of $\mathscr{H}$. Motivated by the situation in Quantum Mechanics and by the dynamical models of Sect. 2 we assume that $G$ has the following two properties:

(I) If $a, b, a^{\prime}, b^{\prime}$ are two pairs of points of $\Phi$ such that $\delta(a, b)=\delta\left(a^{\prime}, b^{\prime}\right)$, then there is a sequence of transformations $g_{n} \in G(n=1,2, \ldots)$ such that $g_{n} a \rightarrow a^{\prime}$ and $g_{n} b \rightarrow b^{\prime}$ as $n \rightarrow \infty$. [This property holds, in particular, if $G \supset S U(\Phi)$.]

(II) Time continuity. For every $g \in G$ there exists a two-parameter family: $t, \tau$ $\rightarrow g_{t \tau} \in G(t, \tau \in[0,1], t \geqq \tau)$ such that: 1) $\left.\left.g_{00}=i d, g_{10}=g, 2\right) g_{t \sigma} g_{\sigma \tau}=g_{t \tau}(t>\sigma>\tau), 3\right)$ the application $t, \tau, x \rightarrow g_{t \tau} x(x \in \Phi, 0 \leqq \tau \leqq t \leqq 1)$ is continuous in the topology of $[0,1] \times[0,1] \times \Phi$.

We shall show that for a semigroup $G$ with the properties I, II, the only mechanism which can prevent the mobility phenomenon is the existence of the following limiting distances.

Definition 1. For an homeomorphism $g: \Phi \rightarrow \Phi$, an angular distance $\alpha \in\left[0, \frac{\pi}{2}\right]$ will be called limiting from below (non-shrinking) if $\delta(a, b)=\alpha \Rightarrow \delta(g a, g b) \geqq \alpha$, and limiting from above (non-expanding) if $\delta(a, b)=\alpha \Rightarrow \delta(g a, g b) \leqq \alpha$ for any $a, b \in \Phi$. The distance $\alpha$ will be called limiting from below (above) for the semigroup $G$ if $\alpha$ is limiting from below (above) for every $g \in G$.

Obviously, for any homeomorphism $g$, the angle 0 is limiting from below as well as from above, while $\frac{\pi}{2}$ is limiting from above. These limiting distances will be called trivial. (However, $\frac{\pi}{2}$, if limiting from below will be classified as non-trivial.) There is the following simple lemma:

Lemma 1. If $G$ is a semigroup of homeomorphisms of $\Phi$ with the properties I, II, then either the phenomenon of mobility takes place (every two points $a \neq b$ can be brought simultaneously to any two open surroundings by elements $g \in G)$, or $G$ has a nontrivial limiting distance on $\Phi$.

Proof. For any $a, b \in \Phi, a \neq b$, denote by $\Delta(a, b)$ the set of all angular distances which can be approached by the distances of the transformed pairs $g a, g b(g \in G)$. Obviously, $\delta(a, b) \in \Delta(a, b)$. By definition, $\Delta(a, b)$ is a closed subset of $\left[0, \frac{\pi}{2}\right]$. Furthermore, $\Delta(a, b)$ has the following simple properties:

(1) If $a^{\prime}, b^{\prime} \in \Phi$ and $\delta\left(a^{\prime}, b^{\prime}\right) \in \Delta(a, b)$, then due to (I) not only the distance $\delta\left(a^{\prime}, b^{\prime}\right)$ can be approached by $\delta(g a, g b)$ but also the pair $a^{\prime}, b^{\prime}$ can be approached by the pairs $g a, g b$ in the topology of $\Phi \times \Phi$.

(2) Due to the continuity of the transformations $h \in G$, this further implies that the distances $\delta\left(h a^{\prime}, h b^{\prime}\right)(h \in G)$ belong to $\Delta(a, b)$. Hence:

$$
\delta\left(a^{\prime}, b^{\prime}\right) \in \Delta(a, b) \Rightarrow \Delta\left(a^{\prime}, b^{\prime}\right) \subset \Delta(a, b) .
$$


As a particular consequence, $\delta\left(a^{\prime}, b^{\prime}\right)=\delta(a, b) \Rightarrow \Delta\left(a^{\prime}, b^{\prime}\right)=\Delta(a, b)$, and so $\Delta(a, b)$ depends only on the distance $\delta(a, b)$ and not on the particular choice of the two points: $\Delta(a, b)=\Delta(\delta(a, b))$.

Due to the continuity property (II) for any $\delta \in\left[0, \frac{\pi}{2}\right], \Delta(\delta)$ is connected. Hence, $\Delta(\delta)$ has to be a closed interval. Now, there are only two possibilities:

- For any $\delta \in\left(0, \frac{\pi}{2}\right], \Delta(\delta)$ coincides with $\left[0, \frac{\pi}{2}\right]$.

- There exists a $\delta, 0<\delta \leqq \frac{\pi}{2}$, such that $\Delta(\delta) \neq\left[0, \frac{\pi}{2}\right]$.

In the first case, consistent with (I), the images of any pair of points can approximate any other pair of points, and the phenomenon of mobility occurs. In the second case both end points of $\Delta(\delta)$ are limiting distances for $G$ and one of them is non-trivial.

To examine the alternative of the "mobility," one is thus lead to study homeomorphisms of the unit spheres which admit limiting distances. The significance of the limiting distance varies from the real to the complex cases. If $\Phi$ is a projective sphere in a real Hilbert space, then any ray $a=\{x\} \in \Phi$ is just a pair of unit vectors $\{x,-x\} \subset S$. The angle $\alpha$ between two rays corresponds to two possible angles between the vectors: $\alpha$ and $\pi-\alpha$. If $\operatorname{dim} \mathscr{H}>2$, for any mapping $g \in G$ (rays into rays) there are two continuous mappings $g_{1}, g_{2}: S \rightarrow S$ (vectors into vectors), such that $g\{x\}=\left\{g_{j} x\right\}(j=1,2)$. If $g$ has a non-trivial limiting distance, so $\operatorname{dog} g_{1}$ and $g_{2}$. If $\operatorname{dim} \mathscr{H}=2$ the same holds due to (II). Hence, the question about the homeomorphisms of the projective sphere with limiting distances reduces to a structurally simpler problem of homeomorphisms of the vector sphere $S$. The complex case does not admit such reduction, and hence, it must be treated separately. In the subsequent study the following general definitions will be employed:

Definition 2. For a mapping $g: \Phi \rightarrow \Phi$ an angle $\alpha$ is called conservative if it is simultaneously limiting from below and from above, i.e., if $\delta(x, y)$ $=\alpha \Rightarrow \delta(g x, g y)=\alpha$.

Definition 3. For any point $x \in \Phi$, where $\Phi$ is a real or complex projective sphere, we define the open and closed $\alpha$-balls $(x)_{\alpha}$ and $[x]_{\alpha}$, the $\alpha$-sphere $\Phi_{\alpha}(x)\left(\alpha \in\left(0, \frac{\pi}{2}\right)\right)$ centered at $x$, and their closed and open exteriors $[x]^{\alpha},(x)^{\alpha}$ by:

$$
\begin{aligned}
(x)_{\alpha}= & \{y \in \Phi: \delta(x, y)<\alpha\}, \quad[x]_{\alpha}=\{y \in \Phi: \delta(x, y) \leqq \alpha\}, \\
\Phi_{\alpha}(x)= & \{y \in \Phi: \delta(x, y)=\alpha\} . \\
& {[x]^{\alpha}=\Phi \backslash(x)_{\alpha}, \quad(x)^{\alpha}=\operatorname{int}[x]^{\alpha}=\Phi \backslash[x]_{\alpha} . }
\end{aligned}
$$

in general, for any subset $Y \subset S$ :

$$
(Y)_{\alpha}=\bigcup_{y \in Y}(y)_{\alpha}, \quad[Y]^{\alpha}=S \backslash(Y)_{\alpha} .
$$

The corresponding open and closed $\alpha$-balls $(x)_{\alpha},[x]_{\alpha}, \alpha$-spheres $S_{\alpha}(x)$, their exteriors $[x]^{\alpha},(x)^{\alpha}$ for the real euclidean sphere $S$ will be obtained just by substituting everywhere $S$ for $\Phi$ and $\gamma$ for $\delta$, and $\pi$ for $\frac{\pi}{2}$. Note that

$$
\begin{gathered}
\left((Y)_{\alpha}\right)_{\beta}=(Y)_{\alpha+\beta}, \\
Y \subset Z \Rightarrow(Y)_{\alpha} \subset(Z)_{\alpha} .
\end{gathered}
$$


Lemma 2. If $g: \Phi \rightarrow \Phi$ is a homeomorphism, and $\alpha \in\left(0, \frac{\pi}{2}\right)$ is a limiting angle, then:

$$
\begin{aligned}
\alpha \text { non-shrinking } \Rightarrow g(x)_{\alpha} \supset(g x)_{\alpha}, \\
\alpha \text { non-expanding } \Rightarrow g(x)_{\alpha} \subset(g x)_{\alpha} .
\end{aligned}
$$

The same holds for the real vector sphere $S$ and limiting angle $\alpha \in(0, \pi)$.

Proof. The proof follows easily from the connectivity of $(x)_{\alpha}$.

\section{A Lemma on Homeomorphisms of the Real Euclidean Spheres}

In an arbitrary (unlimited) metric space, homeomorphisms can exist which admit limiting distances, though they conserve none (an example is a conformal mapping in $\mathbb{R}^{n}$ ). This is not the case of the Hilbertian spheres. Here, one has:

Lemma 3. If $g$ is a homeomorphism of the unit sphere $S$ in a real Hilbert space, admitting a non-trivial limiting angle, then $g$ has also a conservative angle.

Proof. Consider the set $A_{g} \subset[0, \pi]$ of all limiting non-shrinking distances of $g$ (including the trivial one $\alpha=0$ ) and the set $B_{g}$ of all non-expanding distances (including 0 and $\pi$ ). Note that $A_{g}$ and $B_{g}$ are closed in $[0, \pi]$. Furthermore they have the following simple properties:

(1) Additivity. If $\alpha, \beta \in A_{g}$ and $\alpha+\beta \leqq \pi$, then $\alpha+\beta \in A_{g}$. Similarly, $\alpha, \beta \in B_{g}$, $\alpha+\beta \leqq \pi \Rightarrow \alpha+\beta \in B_{g}$

(2) Distributivity. If $\alpha \in A_{g}, \beta \in B_{g}$, and $\alpha \geqq \beta$, then $\alpha-\beta \in A_{g}$.

(3) If $\alpha, \beta \in A_{g}$ and $\alpha+\beta \in B_{g}$, then $\alpha, \beta, \alpha+\beta$ are conservative. The same is true if $\alpha, \beta \in B_{g}, \alpha+\beta \in A_{g}$.

(4) Reflection.

$$
\begin{aligned}
& \alpha \in A_{g}, \quad \alpha \geqq \frac{\pi}{2} \Rightarrow 2(\pi-\alpha) \in B_{g}, \\
& \beta \in B_{g}, \quad \beta \geqq \frac{\pi}{2} \Rightarrow 2(\pi-\beta) \in A_{g} .
\end{aligned}
$$

The proofs of these properties follow from Lemma 2 and elementary properties of the distance on $S$. As an example we shall motivate (4.1). If $\alpha \in A_{g}$, then for any $x \in S$ the open sphere $(x)_{\alpha}$ cannot shrink: $g(x)_{\alpha} \supset(\bar{x})_{\alpha}(\bar{x}=g x)$. This means that, inversely, the closed ball $[x]^{\alpha}$ with the center at $-x$ and radius $\pi-\alpha$ cannot expand: $g[x]^{\alpha} C[\bar{x}]^{\alpha}$. Since $x$ is arbitrary, it means that no sphere with the radius $\pi-\alpha$ can expand: its image is always contained in a certain new sphere with the radius $\pi-\alpha$. As it is not granted that $g$ maps the center into the center, the radius $\pi-\alpha$ is not necessarily limiting, but the diameter $2(\pi-\alpha)$ is. The proof of (4.2) is analogous.

It turns out that the properties (1-4) restrict strongly the structure of the limiting angles. There is:

(5) Suppose, $\alpha>0$ is an element of $A_{g}$. Then either $\alpha$ is conservative, or there exists $\alpha^{\prime} \in A_{g}, 0<\alpha^{\prime}<\alpha$.

Indeed, in view of the additivity (1) all the multiples $n \alpha \leqq \pi$ belong to $A_{g}$. Let $n$ be the greatest natural number for which $n \alpha \leqq \pi[$ and so, $(n+1) \alpha>\pi]$. In view of the reflection property (3), $2 \beta=2(\pi-n \alpha) \in B_{g}$, and $n \alpha \leqq \pi<(n+1) \alpha \Rightarrow 0 \leqq \pi-n \alpha$ $<\alpha \Rightarrow 0 \leqq 2 \beta<2 \alpha$. Now, there are four possibilities. If $2 \beta=0$ then $n \alpha=\pi$ and by 


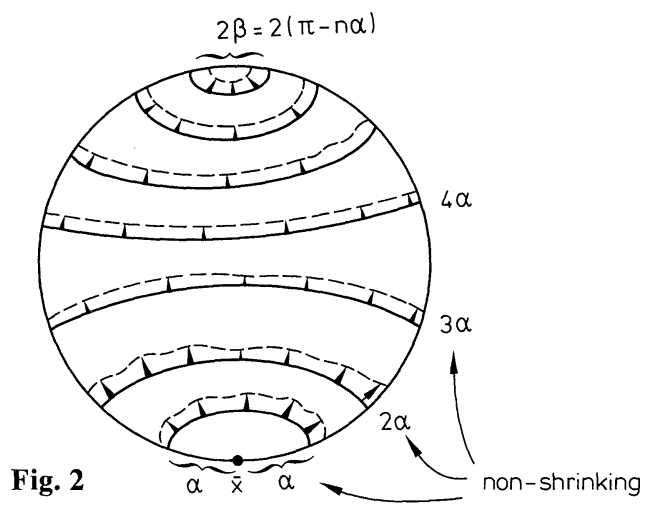

virtue of (3), $\alpha$ is conservative. If $0<2 \beta<\alpha$, then $\alpha^{\prime}=\alpha-2 \beta$ is a new element of $A_{g}$, thus confirming (5). If $2 \beta=\alpha$ then $\alpha \in B_{g}$ in view of (1), and $\alpha$ is conservative. Finally, if $2 \beta>\alpha$, the new angle $\alpha^{\prime} \in A_{g}$ is obtained as $\alpha^{\prime}=2 \alpha-2 \beta$, again ascertaining (5).

The proof of Lemma 3 is now easily concluded. Note, that if $g$ admits at least one non-trivial angle $\alpha \in A_{g} \cup B_{g}$, the $A_{g}$ must contain at least one $\alpha>0$. Indeed, even if we initially assumed only the existence of a non-trivial $\beta \in B_{g}, 0<\beta<\pi$, (4) yields the existence of an angle $\alpha \in A_{g}, 0<\alpha<\pi$. Now, there are only two situations possible. Either among the positive angles $\alpha \in A_{g}$ the smallest one exists: then it is conservative and the lemma is proved. Or the set $A_{g}$ contains positive angles, arbitrarily close to zero. In that case, every angle $\gamma \in(0, \pi)$ can be approximated from below by finite sums of the elements of $A_{g}$. Since $A_{g}$ is additive and closed, every $\gamma \in A_{g}$, and as a consequence of (3) every angle also belongs to $B_{g}$. Hence, every angle is conservative.

\section{Lemma About the Complex Homeomorphisms}

The complex case requires separate treatment. Given a unit spheres $S$ in a complex Hilbert space $\mathscr{H}$ the "angular balls" $\left\{\psi \in \mathscr{H}:\left|\left(\psi, \psi_{0}\right)\right|^{2} \geqq \cos ^{2} \alpha=\right.$ const $\}$ are connected and cannot be reduced to disjoint "vector balls" as in the real case. Yet, the projective complex sphere $\Phi$ too admits a construction similar to that of Lemma 3.

Below we use notation of Definition 3. For any $Z \subset \Phi$, let $Z^{\perp}$ be the set of all rays orthogonal to $Z$. Obviously, $Z=\Phi \backslash(Z)_{\pi / 2}$. Given any two points $a, b \in \Phi$, $a \neq b, \Phi_{a b}=\left(\{a, b\}^{\perp}\right)^{\perp}$ is the two dimensional projective sub-sphere of $\Phi$ spanned by $a$ and $b$. In what follows, a geometric image of the 2-dimensional subspheres is essential. It turns our that the rays of $\Phi_{a b}$ can be represented as the points of the surface of a unit sphere in $\mathbb{R}^{3}$; the orthogonal rays being mapped into the antipodes of the sphere, any two rays $a, b$ with $\delta(a, b)=\alpha$ represented by two surface points whose angular distance in $\mathbb{R}^{3}$ is $\tilde{\alpha}=2 \alpha$. For any $a \in \Phi$ the intersections $(a)_{\alpha} \cap \Phi_{a b}$ and $(a)^{\alpha} \cap \Phi_{a b}$ then are represented by the spherical surroundings with the radii $2 \alpha$ and $\pi-2 \alpha$ centered at $a$ and its antipode $a^{*}$ respectively (Fig. 3). 


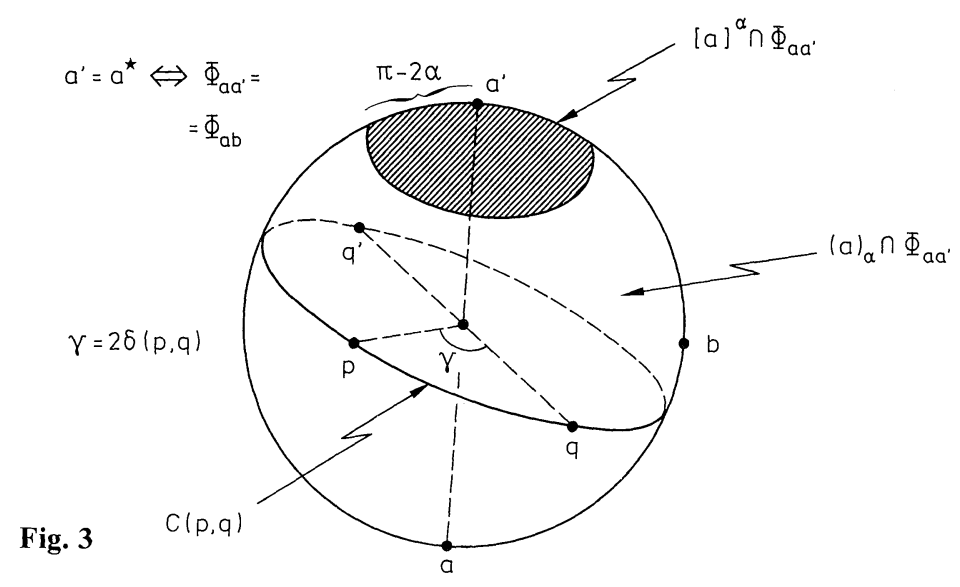

Lemma 4. Let $G$ be a semigroup of homeomorphisms of the projective sphere $\Phi$ in a complex Hilbert space $\mathscr{H}$, obeying (I) and (II) and admitting a non-trivial limiting angle. Then, $G$ has also a non-trivial conservative angle.

Proof. Consider the subsets $A_{G} \subset\left[0, \frac{\pi}{2}\right]$ and $B_{G} \subset\left[0, \frac{\pi}{2}\right]$ of all non-shrinking and all non-expanding limiting distances of the semigroup $G$. As before, one shows that $A_{G}$ and $B_{G}$ are closed. By arguments analogous to those of Lemma 3 one shows the additivity and distributivity properties (1), (2), (3). A difference lies in property (4). Let $\frac{\pi}{2} \leqq \alpha \leqq \frac{\pi}{2}$, and consider $[a]^{\alpha}=\{x \in \Phi: \delta(a, x) \geqq \alpha\}$. This subset is now a "closed ring" in $\Phi$ containing the hyper-sphere $a^{\perp}$. For all $a^{\prime} \in a^{\perp}$, consider the intersections $[a]^{\alpha} \cap \Phi_{a a^{\prime}}$. They are of the form of closed circles in $\Phi_{a a^{\prime}}$ centered around the points $a^{\prime}$, as shown on Fig. 4 . Thus, $[a]^{\alpha}$ is a sum of the family of disjoint closed circles labelled by the elements $a^{\prime} \in a^{\perp}$. As there is no unique isomorphism between any two circles (lying in different $\Phi_{a a^{\prime}}$ subspaces), $[a]^{\alpha}$ is a fiber bundle of the form of a "generalized torus," whose fiber is the circle, and base is the projective sphere $a^{\perp} .^{2}$ ) If $b \neq a,[b]^{\alpha}$ has again circular intersections with $\Phi_{a a^{\prime}}$, though their sizes are varying. Now, put $\beta=\frac{\pi}{2}-\alpha$ and consider two closed domains $[a]^{\alpha}$ and $[b]^{\alpha}$ $(a, b \in \Phi)$. Depending on the angular distance $\delta(a, b)$ there are three topological situations possible:

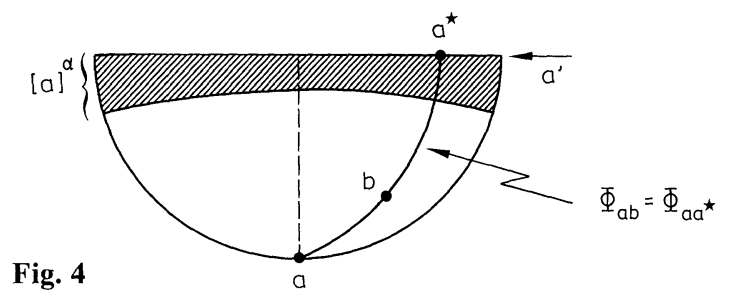

2 The boundary of $[a]^{\alpha}$ is homeomorphic to a real euclidean sphere 


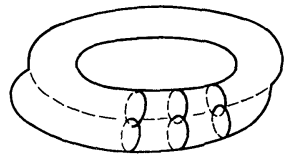

Fig. 5

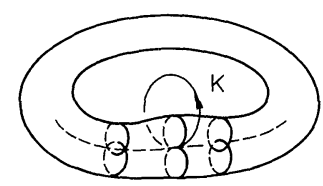

Fig. 6

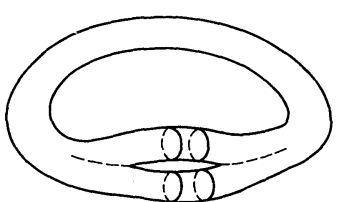

Fig. 7

1) $\delta(a, b)<2 \beta$. For each subspace $\Phi_{a a}\left(a^{\prime} \in a^{\perp}\right)$ the common parts $[a]^{\alpha} \cap \Phi_{a a^{\prime}}$ and $[b]^{\alpha} \cap \Omega_{a a^{\prime}}$ are two intersecting circles. Hence, $[a]^{\alpha}$ and $[b]^{\alpha}$ overlap all over the base space $a^{\perp}$ (Fig. 5).

2) $\delta(a, b)=2 \beta$. The circles defined as $[a]^{\alpha} \cap \Phi_{a a^{\prime}}$ and $[b]^{\alpha} \cap \Phi_{a a^{\prime}}$ intersect in all $\Phi_{a a^{\prime}}$, except the subspace $\Phi_{a b}$, where they are merely tangent. The tangency point $c \in \Phi_{a b}$ is defined as the only point in $[a]^{\alpha} \cap[b]^{\alpha} \cap \Phi_{a b}$, and is topologically distinguished (Fig. 6).

3) $\delta(a, b)>2 \beta$. The $[a]^{\alpha}$ and $[b]^{\alpha}$ partly split. The base space $a^{\perp}$ is now divided into two non-empty, disjoint domains: a closed domain

$$
\Xi=\left\{a^{\prime} \in a^{\perp}:[a]^{\alpha} \cap[b]^{\alpha} \cap \Phi_{a a^{\prime}} \neq \emptyset\right\}
$$

and an open domain $\Omega=a^{\perp} \backslash \Xi$. The fibers of $[a]^{\alpha}$ and $[b]^{\alpha}$ over $\Omega$ are disjoint circles, while the fibers over $\Xi$ are tangent or overlapping (Fig. 7).

Let now $\alpha \in\left(\frac{\pi}{4}, \frac{\pi}{2}\right)$ be a non-expanding angle of $G$, and $a, b \in \Phi$ two points with $\delta(a, b)=2 \beta, \beta=\frac{\pi}{2}-\alpha$. The "double torus" $[a]^{\alpha} \cup[b]^{\alpha}$ is then of type 2) (Fig. 6). Suppose, there is a transformation $g \in G$ yielding $\delta(g a, g b)<2 \beta$. In agreement with the continuity assumption (II) there would exist a two-parameter continuous family $g_{t \tau} \in G(\tau \leqq t)$ such that $g_{00}=i d, g_{10}=g, g_{t \sigma} g_{\sigma \tau}=g_{t \tau}(t \geqq \sigma \geqq \tau)$. Denote $g_{t 0}=g_{t}$. Then consider a closed curve $K$ in $[a]_{\alpha} \cap[b]_{\alpha} \cap \Phi_{a b}$ passing through the tangency point $[a]^{\alpha} \cap[b]^{\alpha} \cap \Phi_{a b}$ and surrounding one of the circles $(a)^{\alpha} \cap \Phi_{a b}$, $(b)^{\alpha} \cap \Phi_{a b}$ (Fig. 6). As $K$ does not intersect $(a)^{\alpha} \cup(b)^{\alpha}$, the transformed loop $K_{t}=g_{t} K$ may not intersect $g_{t}\left[(a)^{\alpha} \cup(b)^{\alpha}\right]=g_{t}(a)^{\alpha} \cup g_{t}(b)^{\alpha}$. As $\alpha$ is non-expanding, Lemma 2 implies:

$$
\begin{aligned}
g_{t}(a)_{\alpha} \subset\left(a_{t}\right)_{\alpha}, \quad g_{t}(b)_{\alpha} \subset\left(b_{t}\right)_{\alpha} & \Rightarrow g_{t}(a)^{\alpha} \supset\left(a_{t}\right)^{\alpha}, \quad g_{t}(b)^{\alpha} \supset\left(b_{t}\right)^{\alpha} \cdot\left(a_{t}=g_{t} a, b_{t}=g_{t} b\right) \\
& \Rightarrow g_{t}(a)^{\alpha} \cup g_{t}(b)^{\alpha} \supset\left(a_{t}\right)^{\alpha} \cup\left(b_{t}\right)^{\alpha} .
\end{aligned}
$$

Hence, $K_{t}$ may not intersect $\left(a_{t}\right)^{\alpha} \cup\left(b_{t}\right)^{\alpha}$. However, this is impossible, since if $\delta(g a, g b)=\delta\left(a_{1}, b_{1}\right)<2 \beta$, the double torus $\left(a_{t}\right)^{\alpha} \cup\left(b_{t}\right)^{\alpha}$ evolving from its topological configuration 2) to 1 ) would have to cut the continuously deformed loop $K_{t}$ (see Appendix.) In view of the contradiction, $\delta(g a, g b)>2 \beta$ and $2 \beta$ is a non-shrinking angle of $G$. The inverse implication $\alpha \in A_{G} \Rightarrow 2 \beta \in B_{G}$ can be obtained just by applying the already proved result to the semigroup $G^{-1}$. Once we have the analogue of the reflection property (4), the proof of Lemma 4 is finished via the property (5), like in the real case. 


\section{Mechanism of Rigidity}

In some metrical spaces, the existence of one conservative distance implies that all the distances are conserved. This is so for the angle $\frac{\pi}{2}$ on the projective sphere $\Phi$. If $\operatorname{dim} \mathscr{H} \geqq 4$, the orthogonality relation $\left(\alpha=\frac{\pi}{2}\right)$, via the lattice structure, determines all other angles in $\Phi$, (henceforth, defining the general transition probability between the pure states).This fact is crucial for Piron's philosophy of quantum logic $[13,14]$, and so, we shall call it the "Piron statement." As a consequence, any homeomorphism $g: \Phi \rightarrow \Phi$ conserving the angle $\frac{\pi}{2}$ is an isometry. It is our aim to show that a similar implication exists for other conservative angles. We shall start from the real case.

Assume for a moment that $S$ is just the 2-dimensional surface of the unit sphere in $\mathbb{R}^{3}$. Suppose, $\alpha \neq 0, \frac{\pi}{2}, \pi$ is a conservative angle. For any two points $x, y \in S, x \neq y$, $\gamma(x, y)<\min \{2 \alpha, 2(\pi-\alpha)\}$, consider the pair $D_{\alpha}(x, y)$ of the $\alpha$-circles passing through $x$ and $y$. They form a lense-shaped figure on $S$ with vertices at $x$ and $y$. We shall call it a diangle $x y$.

Consider now three points $x, y, z \in S$.

Then, between the diangles $x y$ and $y z$ a special relation may occur.

Definition 4. Two diangles $x y$ and $y z$ are vertex tangent at $y$ if each circle of the pair $D_{\alpha}(x, y)$ is tangent to one of the circles of $D_{\alpha}(y, z)$ and vice versa (Fig. 8).

As is easily seen, the diangles $x y, y z$ are vertex-tangent if and only if the points $x, y, z$ lie on one great circle and $\gamma(x, y)=\gamma(y, z)$. Suppose now, that $g: S \rightarrow S$ is a homeomorphism, for which $\alpha$ is a conservative angle. Then, $g$ must map any $\alpha$-circle onto another $\alpha$-circle. An $\alpha$-circle crossing $x, y \in S$ must be transformed onto an $\alpha$-circle crossing $\bar{x}=g x$ and $\bar{y}=g y$. Moreover, two tangent $\alpha$-circles must remain tangent. Thus, each diangle is transformed onto a new diangle, and moreover, the vertex-tangent diangles remain vertex-tangent. Hence, if $x, y, z \in S$ lie on one great circle and are equally spaced: $\gamma(x, y)=\gamma(y, z)<\min \{2 \alpha, 2(\pi-\alpha)\}$, their images must also lie on one great circle and be equally spaced. Now, take any $n$ points $(n>2 \pi / \min \{2 \alpha, 2(\pi-\alpha)\})$ dividing any great circle on $S$ into $n$ equal parts. They have to be transformed into new $n$ equally spaced points on another great circle. This means, that each angle $\frac{2 \pi}{n}$ is conserved. Thus, there are conservative

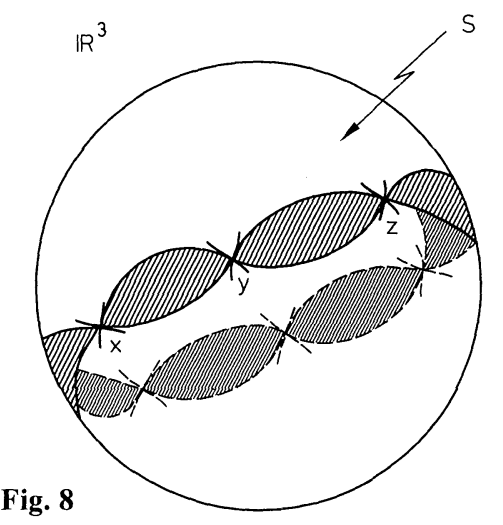


angles arbitrarily close to zero. By additivity and closure of $C_{g}=A_{g} \cap B_{g}$, every angular distance is conserved and $g$ is an isometry.

The above construction has an immediative extension to any real Hilbert space.

Definition 5. Let $S$ be a unit sphere in a real Hilbert space $\mathscr{H}$. For any two points $x, y \in S, x \neq y$, denote by $D_{\alpha}(x, y)$ the family of all $\alpha$-spheres on $S$ which cross $x$ and $y$ :

$$
D_{\alpha}(x, y)=\left\{S_{\alpha}(z): \gamma(z, x)=\gamma(z, y)=\alpha\right\} .
$$

The families $D_{\alpha}(x, y)(x, y \in S)$ owe their structure on the following properties of the $\alpha$-spheres:

Lemma 5. If two $\alpha$-spheres $(0<\alpha<\pi)$ are tangent to the same $\alpha$-sphere at the same point, they are identical.

Lemma 6. If $g: S \rightarrow S$ is a homeomorphism of the real unit sphere, and $\alpha \in[0, \pi]$ is a non-trivial conservative angle of $g$, then $g$ transforms any sphere $S_{\alpha}(x)(x \in S)$ exactly onto $S_{\alpha}(g x)$, its interior $(x)_{\alpha}$ onto the interior $(g x)_{\alpha}$ and the exterior $(x)^{\alpha}$ onto the exterior $(g x)^{\alpha}$.

Lemma 7. If $g$ is a homeomorphism $S \rightarrow S$, and $\alpha \neq 0, \frac{\pi}{2}, \pi$ is a conservative distance on $S$, then each two tangent $\alpha$-spheres on $S$ are mapped onto two tangent $\alpha$-spheres. Moreover, the tangency point is mapped into the tangency point.

Lemma 8. For $x, y \in S, x \neq y, \gamma(x, y)<\min (2 \alpha, 2(\pi-\alpha)), a \neq \frac{\pi}{2}, x$ and $y$ are the unique points contained simultaneously in all the spheres of the family $D_{\alpha}(x, y)$ :

$$
\bigcap D_{\alpha}(x, y)=\{x, y\} \text {. }
$$

Definition 6. Let $x, y, z \in S, x \neq y \neq z$. Two families $D_{\alpha}(c, y)$ and $D_{\alpha}(y, z)$ will be called tangent at $y\left(D_{\alpha}(x, y) \sim D_{\alpha}(y, z)\right)$ if every sphere of the family $D_{\alpha}(x, y)$ is tangent at $y$ to one of the spheres of $D_{\alpha}(y, z)$ and vice versa.

Lemma 9. For $\alpha \neq 0, \frac{\pi}{2}, \pi, D_{\alpha}(x, y)$, and $D_{\alpha}(y, z)$ are tangent at $y$ if and only if

1) $x, y, z$ lie on great circle in $S$;

2) $\gamma(x, y)=\gamma(y, z)$.

Proof. Suppose first that $x, y, z$ lie on one great circle and $\gamma(x, y)=\gamma(y, z)$. Then, consider the mapping $I_{y}$, which to any $\xi \in S, \xi=\lambda y+y_{\perp},\left(y_{\perp} \perp y\right)$, assigns $\tilde{\xi}=I_{y} \xi$ $=\lambda y-y_{\perp}$ (symmetry with respect to $\left.y\right)$. Obviously, $I_{y}$ is unitary and conserves the distances. Hence it maps $\alpha$-spheres onto $\alpha$-spheres. Moreover, $I_{y} y=y, I_{y} x=z$, $I_{y} z=x$. Hence, every $\alpha$-sphere crossing $x$ and $y$ is transformed into an $\alpha$-sphere crossing $y$ and $z$ and vice versa. This means that $I_{y}$ transforms the family $D_{\alpha}(x, y)$ onto $D_{\alpha}(y, z)$ and inversely. Now, $I_{y}$ has a special property: every $\alpha$-sphere $\left(\alpha \neq 0, \frac{\pi}{2}, \pi\right)$ which passes through $y$ is tangent at $y$ to its $I_{y}$-image. Hence, the mapping $I_{y}$ arranges the families $D_{\alpha}(x, y)$ and $D_{\alpha}(y, z)$ into the pairs of $\alpha$-spheres tangent at $y$ and so:

$$
D_{\alpha}(x, y) \sim D_{\alpha}(y, z)
$$


Inversely, suppose we know only that (4.3) holds. Then denote $\tilde{x}=I_{y} x$, and apply the reflection $I_{y}$ to all spheres of the family $D_{\alpha}(x, y)$. One obtains the new family $D_{\alpha}(y, \tilde{x})$, and due to the properties of $I_{y}$ :

$$
D_{\alpha}(x, y) \sim D_{\alpha}(y, \tilde{x}) .
$$

In view of (4.3) and (4.4) each sphere of $D_{\alpha}(y, x)$ is tangent to one of the $\alpha$-spheres of $D_{\alpha}(x, y)$, and that one, in turn, is tangent to one of the spheres of $D_{\alpha}(y, z)$. By Lemma 5 , each sphere of the family $D_{\alpha}(y, \tilde{x})$ coincides with one of the spheres of $D_{\alpha}(y, z)$ and vice versa. Hence, $D_{\alpha}(y, \tilde{x})=D_{\alpha}(y, z) \Rightarrow \bigcap D_{\alpha}(y, \tilde{x})=\bigcap D_{\alpha}(y, z)$, and by Lemma $8,\{y, \tilde{x}\}=\{y, z\} \Rightarrow z=\tilde{x}=I_{y} x$. Now, due to the properties of $I_{y}$, the points $x, y$ and $z=I_{y} x$ lie on one great circle and $\gamma(x, y)=\gamma(y, z)$.

Consider now an arbitrary homeomorphism $g: S \rightarrow S$. Suppose, $\alpha \neq 0, \frac{\pi}{2}, \pi$ is a conservative angle for $g$. By Lemmas 5-7, $g$ has to transform any two families $D_{\alpha}(x, y), D_{\alpha}(y, z)$ onto $D_{\alpha}(\bar{x}, \bar{y})$ and $D_{\alpha}(\bar{y}, \bar{z})(\bar{x}=g x, \bar{y}=g y, \bar{z}=g z)$. Moreover, if $D_{\alpha}(x, y) \sim D_{\alpha}(y, z)$ then $D_{\alpha}(\bar{x}, \bar{y}) \sim D_{\alpha}(\bar{y}, \bar{z})$. Hence, each three points $x, y, z$ lying on one great circle, and $\gamma(x, y)=\gamma(y, z)<\min \{2 \alpha, 2(\pi-\alpha)\}$, are transformed into a new triple $\bar{x}, \bar{y}, \bar{z}$ again on a certain great circle and $\gamma(\bar{x}, \bar{y})=\gamma(\bar{y}, \bar{z})$. By an exact repetition of the argument given for $\mathbb{R}^{3}$ we have:

Lemma 10. If $g: S \rightarrow S$ is a homeomorphism of the unit sphere in a real Hilbert space $\mathscr{H}, \operatorname{dim} \mathscr{H}>2$, and $g$ admits a non-trivial conservative angle $\alpha \neq 0, \frac{\pi}{2}, \pi$, then $g$ is an isometry.

Corollary. The exceptional angles are $\alpha=\frac{\pi}{2}$, when the implication of Lemma 10 holds if $\operatorname{dim} \mathscr{H} \geqq 4$ (Piron theorem), and $\alpha=\pi$ when it does not hold at all. The exceptional dimension is $\operatorname{dim} \mathscr{H}=2$, when $S$ is just a unit circle. On the unit circle classes of homeomorphisms can be easily constructed which preserve one particular angle while changing the others.

Complex Case. In case of the complex projective sphere $\Phi$ the existence of a conservative distance has similar consequences. However, the properties of the $\alpha$-spheres on $\Phi$ are no longer so evident. Our notation is as explained by Definition 3.5. We shall again study the properties of the families $D_{\alpha}(a, b)$ (for $a, b \in \Phi)$. One has:

Lemma 11. Suppose $\alpha \in\left(0, \frac{\pi}{2}\right), \alpha \neq \frac{\pi}{4}$, and $a, b \in \Phi, \delta(a, b)<\min \left\{2 \alpha, 2\left(\frac{\pi}{2}-\alpha\right)\right\}$. Then the intersection of the family $D_{\alpha}(a, b)$ consists exclusively of the pair of points $a$ and $b$. If $\alpha=\frac{\pi}{4}$, the same holds provided that $\operatorname{dim} \mathscr{H} \geqq 3$ (author's notes).

To generalize the constructions from a real $\mathscr{H}$, the concept of tangency has to be extended.

Definition 7. Two $\alpha$-spheres $\Phi_{\alpha}(a), \Phi_{\alpha}(b)(a, b \in \Phi)$ will be called tangent if $0<\alpha \leqq \frac{\pi}{4}$ and $\delta(a, b)=2 \alpha$, and semi-tangent if $\frac{\pi}{4}<\alpha<\frac{\pi}{2}$ and $\delta(a, b)=2\left(\frac{\pi}{2}-\alpha\right)$.

The geometric sense of these concepts differs depending on the value of $\alpha$. If $0<\alpha \leqq \frac{\pi}{4}$ and $\delta(a, b)=2 \alpha$, the surfaces $\Phi_{\alpha}(a)$ and $\Phi_{\alpha}(b)$ possess precisely one point $c$ in common: $c$ lies in the smaller arc of the great circle $C_{a b}$ on the middle of distance between $a$ and $b$. The intersections of $\Phi_{\alpha}(a)$ and $\Phi_{\alpha}(b)$ with $\Phi_{a b}$ are two circles on $\Phi_{a b}$, centered at $a$ and $b$, and tangent at $c$. The intersections of $\Phi_{\alpha}(a), \Phi_{\alpha}(b)$ with any 
2-dimensional sub-sphere which does not contain $c$ are either empty or are two non-overlapping circles. If $\alpha=\frac{\pi}{4}$, the picture changes. Two $\frac{\pi}{4}$-spheres $\Phi_{\pi / 4}(a)$, $\Phi_{\pi / 4}(b)$ are tangent in the sense of Definition 7 if $\delta(a, b)=\frac{\pi}{2}(a \perp b)$. By intersecting them with the 2-dimensional $\Phi_{a b}$-sphere one sees that $\Phi_{\pi / 4}(a)$ and $\Phi_{\pi / 4}(b)$ have the whole equatorial circle in $\Phi_{a b}$ in common. Still, the interiors $(a)_{\pi / 4},(b)_{\pi / 4}$ are disjoint. Hence, $\Phi_{\pi / 4}(a), \Phi_{\pi / 4}(b)$ are tangent in a traditional sense, though the tangency point is not unique. If $\frac{\pi}{4}<\alpha<\frac{\pi}{2}$, the conventional tangency of the spheres $\Phi_{\alpha}(a), \Phi_{\alpha}(b)$ becomes impossible. What can occur then is the semi-tangency, meaning that the generalized tori $[a]^{\alpha}$ and $[b]^{\alpha}$ are in the configuration represented in Fig. 6. The intersections of $\Phi_{\alpha}(a), \Phi_{\alpha}(b)$ with any other $\Phi_{a a^{\prime}} \neq \Phi_{a b}$ are now overlapping circles. The point $c$ is topologically distinguished: it is the only point of $[a]^{\alpha} \cup[b]^{\alpha}$ whose every open surrounding $\Omega$ has a non-singleconnected intersection $\Omega \cap\left[(a)^{\alpha} \cup(b)^{\alpha}\right]$. In what follows, $c$ will be called a semi-tangency point.

As the concept of a topologically distinguished point is homeomorphisminvariant, one has the following lemma:

Lemma 12. If $g: \Phi \rightarrow \Phi$ is a homeomorphism of the projective unit sphere $\Phi$, and $\alpha \in\left(0, \frac{\pi}{2}\right)$ is a conservative angle of $g$, then every $\alpha$-sphere $\Phi_{\alpha}(a)$ is mapped onto $\Phi_{\alpha}(\bar{a})$ $(\bar{a}=g a)$, the interior $(a)_{\alpha}$ is mapped onto the interior $(\bar{a})_{\alpha}$ and the exterior $(a)^{\alpha}$ into the exterior $(\bar{a})^{\alpha}$. Moreover, the tangent (or semi-tangent) spheres remain tangent (semi-tangent) and the tangency (semi-tangency) points are mapped onto the tangency points (semi-tangency points).

We note also the following

Lemma 13. If $\Phi_{\alpha}(a)$ is any $\alpha$-sphere $\left(\alpha \in\left(0, \frac{\pi}{2}\right)\right)$ and $c \in \Phi_{\alpha}(a)$, then there is precisely one $\alpha$-sphere tangent (or semi-tangent) to $\Phi_{\alpha}(a)$ at $c$.

We can now apply the previous techniques of describing the triples of points via the families $D_{\alpha}(a, b)$.

Definition 8. Given three points $a, c, b \in \Phi, a \neq b \neq c$;

$$
\delta(a, b), \delta(b, c)<\min \left\{2 \alpha, 2\left(\frac{\pi}{2}-\alpha\right)\right\},
$$

the families of the $\alpha$-spheres $D_{\alpha}(a, c)$ and $D_{\alpha}(c, b)$ will be called tangent at $c\left(D_{\alpha}(a, c)\right.$ $\left.\sim D_{\alpha}(c, b)\right)$ if every sphere of the family $D_{\alpha}(a, c)$ is tangent (or semi-tangent) at $c$ to one of the spheres of $D_{\alpha}(c, b)$ and vice-versa.

Lemma 14. The three points $a, c, b \in \Phi$, with $\delta(a, c), \delta(c, b)<\min \left\{2 \alpha, 2\left(\frac{\pi}{2}-\alpha\right)\right\}$, lie on one great circle and are equally spaced: $\delta(a, c)=\delta(c, b)$, if and only if $D_{\alpha}(a, c)$ $\sim D_{\alpha}(c, b)$. Exception: for $\operatorname{dim} \mathscr{H}=2$ this holds if $a \neq \frac{\pi}{4}$.

Proof. The proof uses the symmetry $I_{c}$ with respect to the point $c$ and Lemmas 11-13 exactly as in the real case.

Suppose now that $g: \Phi \rightarrow \Phi$ is a homeomorphism of $\Phi$ admitting a conservative distance $\alpha \in\left(0, \frac{\pi}{2}\right)$. In view of Lemma $12, g$ must transform any two tangent families of $\alpha$-spheres, $D_{\alpha}(a, c) \sim D_{\alpha}(c, b)$, into another two tangent families: $D_{\alpha}(\bar{a}, \bar{c})$ $\sim D_{\alpha}(\bar{c}, \bar{b})(\bar{a}=g a, \bar{b}=g b, \bar{c}=g c)$. Thus, if the triple $a, c, b$ was equally spaced on a certain great circle, so is the transformed triple $\bar{a}, \bar{b}, \bar{c}$. If $\alpha=\frac{\pi}{4}$, this conclusion holds 
provided that $\operatorname{dim} \mathscr{H}>2$. By arguments identical to those for the real case, $g$ must conserve any angular distance. Summarizing both real and complex cases we have:

Theorem I. If $g$ is a homeomorphism of the projective sphere $\Phi$ in a Hilbert space $\mathscr{H}$ over real numbers, with $\operatorname{dim} \mathscr{H}>2$, or over the complex numbers, and $g$ admits at least one conservative distance $\alpha \neq 0, \frac{\pi}{4}, \frac{\pi}{2}$, then $g$ is an isometry. In the real case an exceptional angle is $\frac{\pi}{2}$, for which the conclusion holds only if $\operatorname{dim} \mathscr{H} \geqq 4$ (case of Piron). In the complex case there are two exceptional angles $\alpha=\frac{\pi}{4}$ and $\alpha=\frac{\pi}{2}$, for which the conclusion holds if $\operatorname{dim} \mathscr{H} \geqq 3$ and $\operatorname{dim} \mathscr{H} \geqq 4$ respectively.

The above theorem is in fact an extension of the statement of Piron to $\alpha \neq \frac{\pi}{2}$. It may be also viewed as a generalization of Wigner's theorem, starting that a homeomorphism of $\mathscr{H}$ conserving the norms and angular distances can be reduced to either unitary or anti-unitary transformation [15]. Due to Theorem I, the same can now be ascertained for a homeomorphism of the unit sphere which has only one non-trivial conservative distance.

\section{Theorem of Mobility}

Within assumptions of Lemmas 3 and 4, the appearance of a conservative distance on $\Phi$ is a consequence of the existence of a limiting distance, and that one, in turn, is the only alternative of the mobility phenomenon. Hence, one arrives at the following theorem, which states that for the semigroups with the properties I, II "complete fluidity" is the only escape from "complete rigidity."

Theorem II (Theorem of Mobility). Suppose, G is a semigroup endowed with the properties (I) and (II) of homeomorphisms of the projective sphere $\Phi$ in a real or complex Hilbert space $\mathscr{H}, \operatorname{dim} \mathscr{H} \geqq 4$. Then, if $G$ contains some transformations which are not isometries, the phenomenon of mobility takes place: each two points $a, b \in \Phi, a \neq b$, can be arbitrarily approached or arbitrarily separated, by operations $g \in G$ bringing them simultaneously to two arbitrary vicinities on $\Phi$.

The exceptions to this theorem exist for $\operatorname{dim} \mathscr{H}=2,3$, and are due to the limitations of Theorem I. One of exceptional dynamical models, with $\operatorname{dim} \mathscr{H}=2$, where an incomplete mobility coexists with the conservative angle $\frac{\pi}{2}$, is represented by Eqs. (2.2). However, no such models can be constructed for $\operatorname{dim} \mathscr{H}>3$.

A technical consequence of Theorem II is non-existence of any non-trivial continuous function $f(a, b)(a, b \in \Phi)$ which would be invariant under all transformations $g \in G$, thus generalizing the quantum mechanical quantity $|(\psi, \phi)|^{2}$. Some conceptual consequences are also imminent. They parallel the idea that the axioms about the nature of a physical system cannot be postulated, without consideration of the consistency with the dynamical laws (Haag). One of the main axioms characterizing the quantum theory is the impossibility of infinitely selective measurements (Dirac), and the related complementarity doctrine (Bohr). Simplified ad extremis they just tell that for any two unit vectors $\psi, \phi \in \mathscr{H}$, representing the states of a microsystem, with $|(\psi, \phi)|^{2} \neq 0$, no measuring device can be found which would accept all particles in the state $\psi$ while simultaneously rejecting all particles in the state $\phi$. This is, in fact, the simplest 
formulation of the uncertainty principle (see our discussions in [6]). Now, due to Theorem II, in a non-linear dynamics with the properties (I) and (II), this principle must be broken. Given any two states $a=\{\psi\}, b=\{\phi\}, a \neq b$, an arbitrarily selective measurement can be arranged with the help of dynamical separation operations assured by Theorem II. It is enough to perform first any dynamical operations $g \in G$ which brings $a$ and $b$ to vicinities of any two orthogonal states, and then to carry out an ordinary quantum mechanical filtering. Hence, in a nonlinear dynamics with the properties (I) and (II) the "non-linear wave packets" acquire some features of classical systems (see also discussions in $[8,12]$ ).

\section{Appendix}

Proof of Lemma 4 (Detail). The proof of the impossibility of the transition through the sequence of configurations on Fig. $7 \rightarrow 6 \rightarrow 5$ requires the construction of mappings which provide two-dimensional images of $g_{t}[a]^{\alpha}$ and $g_{t}[b]^{\alpha}$. For any $a \in \Phi$ the sphere $\Phi$ splits into a continuous family of 2-dimensional subspaces $\Phi_{a a^{\prime}}$ $\left(a^{\prime} \in a^{\perp}\right)$ which intersect only at $a$; we shall refer to them as to "leaves." Each leaf $\Phi_{a a^{\prime}}$ is isomorphic to the sphere in $\mathbb{R}^{3}$ represented on Fig. 3, with the angular distances $\tilde{\alpha}, \widetilde{\beta}, \ldots$ doubled comparing to those on $\Phi$, and with $a$ and $a^{\prime}$ playing the role of two opposite "poles." Given $b \neq a$, the leaf containing $b$ is labelled as: $\Phi_{a b}=\Phi_{a a^{*}}\left(a^{*} \in a^{\perp}\right)$. On this leaf the antipode of any point $c$ will be denoted $c^{*}$. The intersection of any leaf $\Phi_{a a^{\prime}}$ with $[a]^{\alpha}$ is the closed circle on $\Phi_{a a^{\prime}}$ of radius $\tilde{\beta}=\pi-\tilde{\alpha}$ centered at $a^{\prime}$. The intersections $[b]^{\alpha} \cap \Phi_{a a^{\prime}}$ vary. If $a^{\prime}=a^{*}, \Phi_{a a^{*}} \cap[b]^{\alpha}$ is the $\widetilde{\beta}$-circle centered at $b^{*}$. If $a^{\prime} \neq a^{*}, \Phi_{a a^{\prime}} \cap[b]^{\alpha}$ is a bigger circle, centered at the antipode $b_{\|}^{\prime}$ of the point $b_{\|}$obtained as the normalized projection of $b=\{\varphi\}$ onto $\Phi_{a a^{\prime}}$; its radius $\widetilde{\beta}^{\prime}$ and the distance $\tilde{\gamma}^{\prime}$ of the center $b_{\|}^{\prime}$ from $a^{\prime}$ given by:

$$
\sin ^{2} \frac{\tilde{\beta}^{\prime}}{2}=\frac{1}{|\lambda|^{2}} \sin ^{2} \frac{\tilde{\beta}}{2}, \quad \cos ^{2} \frac{\tilde{\gamma}^{\prime}}{2}=\frac{1}{|\lambda|^{2}} \cos ^{2} \frac{\tilde{\gamma}}{2},
$$

where $|\lambda|^{2}$ expressed the norm decrease due to the projection $\varphi \rightarrow \varphi_{\|}$of $\varphi$ onto $\Phi_{a a^{\prime}}$ :

$$
|\lambda|^{2}=\left\|\varphi_{\|}\right\|^{2}=1-\sin ^{2} \frac{\tilde{\gamma}}{2} \sin ^{2} \delta\left(a^{\prime}, \tilde{a}\right)<1 \Rightarrow \tilde{\gamma}^{\prime}<\tilde{\gamma}, \quad \tilde{\beta}^{\prime}>\tilde{\beta} .
$$

The mapping $H_{a b}: \Phi_{a a^{\prime}} \rightarrow \Phi_{a b}=\Phi_{a a^{*}}$ is now defined for any $a^{\prime} \in a^{\perp}, a^{\prime} \notin b^{\perp}$ by three demands: 1) $H_{a b}$ is an isometry, 2) $\left.H_{a b} a=a, H_{a b} a^{\prime}=a^{*}, 3\right) H_{a b} b_{\|}=c$, where $c$ is the point of $\Phi_{a a^{*}}$ lying on the great arc $a b$ at the same distance $\tilde{\gamma}^{\prime}$ from $a$ as $b_{\|}$. The $H_{a b}$-pictures of the circles $[a]^{\alpha} \cap \Phi_{a a^{\prime}}$ are all identical and coincide with the same $\widetilde{\beta}$-circle on $\Phi_{a a^{*}}$ centered at $a^{*}$. The images of $[b]^{\alpha} \cap \Phi_{a a^{\prime}}$ are varying. Yet, if $a^{\prime}$ is close to $a^{*}$, they fulfill:

(i) Intersection Property. For all $a, b \in \Phi$ with $\tilde{\gamma}=2 \delta(a, b) \in[\widetilde{\beta}, 2 \widetilde{\beta}]$, there exists a common number $\delta_{0}>0$ such that the $H_{a b}$-pictures of all the circles $[b]^{\alpha} \cap \Phi_{a a^{\prime}}$ with $\delta\left(a^{\prime}, a^{*}\right)<\delta_{0}$ contain the same closed circular surrounding $U[\tilde{\beta} / 2] \subset \Phi_{a b}$ $\cap[b]^{\alpha}$, of the radius $\widetilde{\beta} / 2$, internally tangent to $\Phi_{a b} \cap[b]^{\alpha}$ at its point of minimal distance from $a^{*}$ (authors notes.) In what follows, the open interior of $U[\tilde{\beta} / 2]$ is denoted $U(\widetilde{\beta} / 2)$. If $\tilde{\gamma}=2 \widetilde{\beta}$, the circles $U[\widetilde{\beta} / 2]$ and $[a]^{\alpha} \cap \Phi_{a b}$ are tangent, and $U(\widetilde{\beta} / 2)$, $(a)^{\alpha} \cap \Phi_{a b}$ are disjoint, and if $\tilde{\gamma}<2 \widetilde{\beta}, U(\widetilde{\beta} / 2)$ and $(a)^{\alpha} \cap \Phi_{a b}$ overlap. 
Assume now, $\alpha \in\left(\frac{\pi}{4}, \frac{\pi}{2}\right)$ is a non-expanding angle of

$$
G \Rightarrow g[x]^{\alpha} \supset[g x]^{\alpha} \quad(x \in \Phi, g \in G) .
$$

Then take $a_{0}, b_{0} \in \Phi$ with $\delta\left(a_{0}, b_{0}\right)=2 \beta, \beta=\frac{\pi}{2}-\alpha$. Suppose, $g \in G$ yields $\delta\left(g a_{0}, g b_{0}\right)<2 \beta$, and let $t, \tau \rightarrow g_{t \tau} \in G$ be a continuous family assured by (II), with $g_{10}=g$. Hence, for $a_{t}=g_{t 0} a_{0}, b_{t}=g_{t 0} b_{0}$, the distance $\delta\left(a_{t}, b_{t}\right)$ would decrease continuously from $\delta\left(a_{0}, b_{0}\right)=2 \beta$ to $\delta\left(a_{1}, b_{1}\right)<2 \beta$. Let $\tau$ be the greatest number in $[0,1]$ for which $\delta\left(a_{\tau}, b_{\tau}\right)=2 \beta$. Put $a=a_{\tau}, b=b_{\tau}$, and $h_{t}=g_{t \tau}$. Thus: $a_{t}=h_{t} a, b_{t}=h_{t} b$ $(t \geqq \tau)$. There holds:

(ii) Embedding Property. There is a number $\mu>0$ such that if $t \in[\tau, \tau+\mu)$ then: 1) $\delta\left(a_{t}, b_{t}\right) \in(\beta, 2 \beta]$, and 2) the $h_{t}$-images of $[a]^{\beta} \cap \Phi_{a b}$ belong to the leaves $\Phi_{a_{t} a^{\prime}}$ in the vicinity of $\Phi_{a_{t} b_{t}}=\Phi_{a_{t} b_{b}^{*}}$, with $\delta\left(a^{\prime}, a_{t}^{*}\right)<\delta_{0}$.

Proof. The existence of $\mu_{1}>0$ such that

$$
\tau<t<\tau+\mu_{1} \Rightarrow \delta\left(a_{t}, b_{t}\right) \in(\beta, 2 \beta)
$$

is due to the continuity of $\delta\left(a_{t}, b_{t}\right)$. Now, there also exist a $\mu_{2}>0$ such that for $\tau<t$ $<\tau+\mu_{2}$ and $x \in \Phi_{a b} \cap[a]^{\beta}$ the image $h_{t} x \in \Phi_{a_{t} a^{\prime}}$ where $\delta\left(a^{\prime}, a_{t}^{*}\right)<\delta_{0}$. Indeed, if no such $\mu_{2}$ existed, there would be a sequence of numbers $t_{n} \rightarrow \tau, t_{n} \in(\tau, 1)$, and points $x_{n} \in \Phi_{a b} \cap[a]^{\beta}$ such that $h_{t_{n}} x_{n} \in \Phi_{a_{t_{n} a_{n}^{\prime}}}$ with $\delta\left(a_{n}^{\prime}, a_{t n}^{*}\right)>\delta_{0}$. This would imply that the distances between $h_{t_{n}} x_{n}$ and the compact subsets $\Phi_{a_{t_{n}} b_{n}} \cap\left[a_{t_{n}}\right]^{\beta}$ are limited from below:

$$
\delta\left(h_{t_{n}} x_{n},\left[a_{t_{n}}\right]^{\beta} \cap \Phi_{a_{t_{n}} b_{t_{n}}}\right)>\sigma_{0}>0,
$$

where $\sigma_{0}$ is taken from an elementary calculation (authors notes):

$$
\cos ^{2} \sigma_{0}=1-\sin ^{2} \beta \sin ^{2} \delta_{0} .
$$

On the other hand, as $\Phi_{a b}$ is compact, the sequence $x_{n}$ would possess a convergent subsequence $x_{k_{n}} \rightarrow x_{0} \in \Phi_{a b} \cap[a]^{\beta}$. After substituting $k_{n}$ for $n$ in (A.4) and taking the limit, in view of (II) one should obtain: $\delta\left(x_{0},[a]^{\beta} \cap \Phi_{a b}\right)>\sigma_{0}>0$ which is impossible, as $x_{0} \in[a]^{\beta} \cap \Phi_{a b}$. The existence of $\mu_{2}$ proved, the required $\mu$ is obtained, $\mu=\min \left(\mu_{1}, \mu_{2}\right)$.

Consider now the contour $K \subset \Phi_{a b} \cap[a]^{\beta} \cap[a]_{\alpha} \cap[b]_{\alpha}$ (Fig. 6), enclosing the open circle $(a)^{\alpha} \cap \Phi_{a b}$ and crossing the tangency point $[a]^{\alpha} \cap[b]^{\alpha} \cap \Phi_{a b}$. Apply $h_{t}: \Phi \rightarrow \Phi$. The points $a, b$ will drift into $a_{t}, b_{t}$ with $\delta\left(a_{t}, b_{t}\right)<2 \beta(t>\tau)$. The transformed loop $h_{t} K$, in general, may stick out of the new leaf $\Phi_{a_{t} b_{t}}=\Phi_{a_{t} a}$, but as long as $t \in[\tau, \tau+\mu]$, it is embedded in the collection of nearby leaves $\Phi_{a_{t} a^{\prime}}$ with $\delta\left(a^{\prime}, a_{t}^{*}\right)<\delta_{0}\left(a^{\prime} \in a_{t}^{\perp}\right)$. Then consider $K_{t}=H_{a_{t} b_{t}}\left(h_{t} K\right)$ (the image of the deformed curve on the leaf $\Phi_{a_{t} b_{t}}$ ). Due to (A.3) and intersection property (i), $K_{t}$ must remain outside of the two open circles: $\left(a_{t}\right)^{\alpha} \cap \Phi_{a_{t} b_{t}}$ and $U_{t}(\widetilde{\beta} / 2) \subset\left(b_{t}\right)^{\alpha} \cap \Phi_{a_{t} b_{t}}$. However, if $\delta\left(a_{t}, b_{t}\right)<2 \beta$, it cannot be, as $\left(a_{t}\right)^{\alpha} \cap \Phi_{a_{t} b_{t}}$ and $U_{t}(\widetilde{\beta} / 2)$ overlap:

Fig. 9
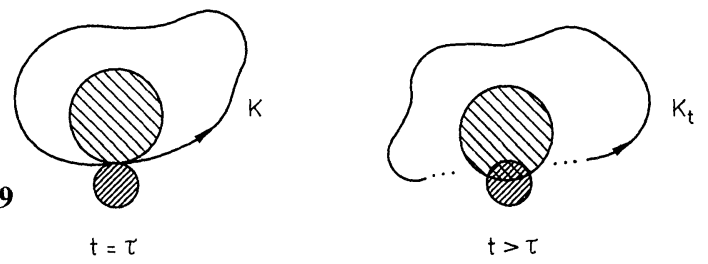
Hence, the assumption that $\delta(a, b)=2 \beta$, but $\delta\left(a_{t}, b_{t}\right)<2 \beta(t>\tau)$ leads to an elementary topological impossibility involving a loop and two moving circles on the 2-dimensional surface of the sphere (Fig. 9).

Acknowledgement. The author is grateful to Professor Augusto Garciá, Professor Jerzy Plebanski and Professor Héctor O. Nava Jaimes for their kind hospitality in CINVESTAV, México, 1983. Thanks also to colleagues from the Departamento de Fisica for their encouragement and helpful discussions.

\section{References}

1. Ruelle, D.: Applications conservant une mesure absolument continue par rapport à $d x$ sur $[0,1]$. Commun. Math. Phys. 55, 47 (1977)

2. Eckmann, J.-P.: Roads to turbulence in dissipative dynamical systems. Rev. Mod. Phys. 53, 643 (1981)

3. Piña, E.: Talk at the Phys. Dept., CINVESTAV, México (1983)

4. Haag, R., Kastler, D.: An algebraic approach to quantum field theory. J. Math. Phys. 5, 848 (1964)

5. Lamb, W.E.: Phys. Today, 22, No. 4, 23 (1969)

6. Mielnik, B.: Generalized quantum mechanics. Commun. Math. Phys. 37, 221 (1974)

7. Lubkin, E.: A physical system which can be forced to execute an arbitrary unitary transformation, and its use to perform arbitrary tests. J. Math. Phys. 15, 673 (1974)

8. Mielnik, B.: Mobility of nonlinear systems. J. Math. Phys. 21, 44 (1980)

9. Waniewski, J.: "Globalna dynamika i Struktura wypukła stanów $w$ teoriach kwantowych i klasycznych", Warszawa 1983 (manuscript of $\mathrm{Ph}$. D. thesis)

10. Waniewski, J.: "Time inversion and mobility of many particle systems. Commun. Math. Phys. 76, $27(1980)$

11. Mielnik, B.: Global mobility of Schrödinger's particle. Rep. Math. Phys. 12, 331 (1977)

12. Haag, R., Bannier, U.: Comments on Mielnik's generalized (non-linear) quantum mechanics. Commun. Math. Phys. 60, 1 (1978)

13. Piron, C.: Axiomatique Quantique. Helv. Phys. Acta 37, 439 (1964)

14. Piron, C.: Foundations of quantum physics. New York: Benjamin 1976

15. Wigner, E.P.: Ann. Math. 40, 149 (1939)

Communicated by R. Haag

Received April 10, 1984; in revised form April 15, 1985 
